\title{
On the Hardness of the Noncommutative Determinant
}

\author{
V. Arvind and Srikanth Srinivasan \\ The Institute of Mathematical Sciences \\ C.I.T. Campus, Chennai 600 113, India \\ \{arvind, srikanth\}@imsc.res.in
}

August 30, 2021

\begin{abstract}
In this paper we study the computational complexity of computing the noncommutative determinant. We first consider the arithmetic circuit complexity of computing the noncommutative determinant polynomial. Then, more generally, we also examine the complexity of computing the determinant (as a function) over noncommutative domains. Our hardness results are summarized below:

- We show that if the noncommutative determinant polynomial has small noncommutative arithmetic circuits then so does the noncommutative permanent. Consequently, the commutative permanent polynomial has small commutative arithmetic circuits.

- For any field $\mathbb{F}$ we show that computing the $n \times n$ permanent over $\mathbb{F}$ is polynomial-time reducible to computing the $2 n \times 2 n$ (noncommutative) determinant whose entries are $O\left(n^{2}\right) \times O\left(n^{2}\right)$ matrices over the field $\mathbb{F}$.

- We also derive as a consequence that computing the $n \times n$ permanent over nonnegative rationals is polynomial-time reducible to computing the noncommutative determinant over Clifford algebras of $n^{O(1)}$ dimension.

Our techniques are elementary and use primarily the notion of the Hadamard Product of noncommutative polynomials.
\end{abstract}

\section{Introduction}

In his seminal paper N91 Nisan first systematically studied the problem of proving lower bounds for noncommutative computation. The focus of his study was noncommutative arithmetic circuits, noncommutative arithmetic formulas and noncommutative algebraic branching programs. In his central result based on a rank argument, Nisan shows that the noncommutative permanent or determinant polynomials in the ring $\mathbb{F}\left\langle x_{11}, \cdots, x_{n n}\right\rangle$ require exponential size noncommutative algebraic branching programs.

Nisan's results are over the free noncommutative ring $\mathbb{F}\langle X\rangle$. Chien and Sinclair, in [CS04, explore the same question over other noncommutative algebras. They refine Nisan's rank argument to show 
exponential size lower bounds for formulas computing the permanent or determinant over specific noncommutative algebras, like the algebra of $2 \times 2$ matrices over $\mathbb{F}$, the quaternion algebra, and a host of other examples.

However, the question of whether there is a small noncommutative circuit for the determinant or permanent remains unanswered. (Indeed, no explicit lower bounds are known for the general noncommutative circuit model.) Since the existence of small noncommutative arithmetic circuits for the permanent would imply the existence of small commutative arithmetic circuits for the permanent, we have a good reason to believe that the permanent does not have small noncommutative arithmetic circuits. However, as far as we know, no such argument has been given for the case of the noncommutative determinant. Indeed, since Nisan [N91] has also shown an exponential separation between the power of noncommutative formulas and circuits, it may very well be that the noncommutative determinant has polynomial-sized arithmetic circuits.

Another motivation for studying the computational difficulty of computing the noncommutative determinant (as a function) is an approach to designing randomized approximation algorithms for the $0-1$ permanent by designing good unbiased estimators based on the determinant. This approach has a long history starting with GG81, KKL+93. Of specific interest are the works of Barvinok [B]; Chien, Rasmussen, and Sinclair [CRS03]; and more recently that of Moore and Russell [MR09. Barvinok B] defines a variant of the noncommutative determinant called the symmetrized determinant and shows that given inputs from a constant dimensional matrix algebra, the symmetrized determinant over these inputs can be evaluated in polynomial time. He uses these to define a series of algorithms that he conjectures might yield progressively better randomized approximation algorithms for the (commutative) permanent. Chien, Rasmussen, and Sinclair CRS03. show that efficient algorithms to compute the determinant over Clifford algebras of polynomial dimension would yield efficient approximation algorithms for the permanent. Moore and Russell [MR09] provide evidence that Barvinok's approach might not work, but their results also imply that computing the symmetrized or standard noncommutative determinant over polynomial dimensional matrix algebras would give a good estimator for the permanent.

\section{Our results}

1. We provide evidence that the noncommutative determinant is hard. We show that if the noncommutative determinant 11 can be computed by a small noncommutative arithmetic circuit, then so can the noncommutative permanent and therefore, the commutative permanent has small commutative arithmetic circuits. This is in marked contrast to the commutative case, where the determinant is known to be computable by polynomial sized circuits, but the permanent is not known (or expected) to have subexponential sized arithmetic circuits.

2. We show that computing the noncommutative determinant over matrix algebras of polynomial dimension is as hard as computing the commutative permanent. We also derive as a consequence that computing the $n \times n$ permanent over nonnegative rationals is polynomial-time reducible to computing the noncommutative determinant over Clifford algebras of $\operatorname{poly}(n)$ dimension.

\footnotetext{
${ }^{1}$ We haven't defined this polynomial formally yet and there are, in fact, many ways of doing it. See Section 2.
} 
This points to the intractability of carrying over Barvinok's approach for large dimension, and also to the possibility that the approach of Chien, Rasmussen, and Sinclair might be computationally infeasible.

We stress that our result here is potentially more useful than a noncommutative circuit lower bound for the determinant, from an algorithmic point of view. For, an arithmetic circuit lower bound result would not rule out the possibility of a polynomial-time algorithm for the noncommutative determinant over even polynomial dimension matrix algebras. For example, Barvinok's algorithm [B] computes the symmetrized determinant over constant dimensional matrix algebras, whereas any algebraic branching program that computes the symmetrized determinant over constant dimensional matrix algebras must be of exponential size [CS04.

\section{Preliminaries}

For any set of variables $X$, let $\mathbb{F}\langle X\rangle$ denote the ring of noncommuting polynomials over $X$. Let $\mathcal{M}(X)$ denote the set of noncommutative monomials over $X$; given $d \in \mathbb{N}$, let $\mathcal{M}_{d}(X)$ denote the monomials over $X$ of degree exactly $d$. For $f \in \mathbb{F}\langle X\rangle$ and $m \in \mathcal{M}(X)$, we will denote by $f(m)$ the coefficient of the monomial $m$ in $f$.

For any ring $R$, we use $M_{n}(R)$ to denote the ring of $n \times n$ matrices with entries from $R$.

Fix $X=\left\{x_{1}, x_{2}, \ldots, x_{m}\right\}$ and $Y=\left\{y_{1}, y_{2}, \ldots, y_{n}\right\}$, two disjoint sets of variables. Given $f \in \mathbb{F}\langle X\rangle$, matrices $A_{i} \in M_{k}(\mathbb{F}\langle Y\rangle)$ for $1 \leq i \leq m$, and $i_{0}, j_{0} \in[k]$, we use $f\left(A_{1}, A_{2}, \ldots, A_{m}\right)\left(i_{0}, j_{0}\right)$ to denote the $\left(i_{0}, j_{0}\right)$ th entry of the matrix $f\left(A_{1}, A_{2}, \ldots, A_{m}\right) \in M_{k}(\mathbb{F}\langle Y\rangle)$.

\subsection{Noncommutative determinants and permanents}

Given $X=\left\{x_{i j} \mid 1 \leq i, j \leq n\right\}$ for $n \in \mathbb{N}$, we define the $n \times n$ noncommutative determinant and permanent polynomials over the set of variables $X$. By fixing the order of multiplication in each monomial of the commutative determinant/permanent polynomials in different ways, one can obtain many different reasonable ways of defining the $n \times n$ noncommutative determinant and permanent,

and indeed many of these definitions have been studied (see [A96], which surveys various flavours of the noncommutative determinant). The most straightforward definitions are those of the Cayley determinant and Cayley permanent - we will denote these by $\operatorname{Cdet}_{n}(X)$ and $\operatorname{Cperm}_{n}(X)$ respectively - which use the row order of multiplication. That is,

$$
\begin{aligned}
\operatorname{Cdet}_{n}(X) & =\sum_{\sigma \in S_{n}} \operatorname{sgn}(\sigma) x_{1, \sigma(1)} \cdot x_{2, \sigma(2)} \cdots x_{n, \sigma(n)}, \\
\operatorname{Cperm}_{n}(X) & =\sum_{\sigma \in S_{n}} x_{1, \sigma(1)} \cdot x_{2, \sigma(2)} \cdots x_{n, \sigma(n)} .
\end{aligned}
$$

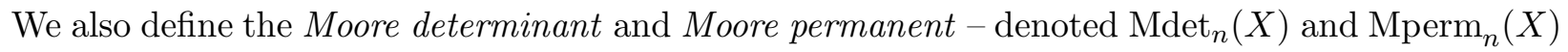
respectively - by ordering the variables in each monomial using the cyclic order of the 
corresponding permutation. Given $\sigma \in S_{n}$, we write it as a product of disjoint cycles $\left(n_{11}^{\sigma} \cdots n_{1 l_{1}}^{\sigma}\right)\left(n_{21}^{\sigma} \cdots n_{2 l_{2}}^{\sigma}\right) \cdots\left(n_{r 1}^{\sigma} \cdots n_{r l_{r}}^{\sigma}\right)$ such that $\forall i \in[r]$ and $j \in\left[l_{r}\right] \backslash\{1\}$, we have $n_{i 1}^{\sigma}<n_{i j}^{\sigma}$ and $n_{11}^{\sigma}>n_{21}^{\sigma}>\cdots>n_{r 1}^{\sigma}$. The Moore determinant and permanent are defined as

$$
\begin{aligned}
\operatorname{Mdet}_{n}(X) & =\sum_{\sigma \in S_{n}} \operatorname{sgn}(\sigma) x_{n_{11}^{\sigma}, n_{12}^{\sigma}} \cdots x_{n_{1 l_{r}}^{\sigma}, n_{11}^{\sigma}} \cdots x_{n_{r 1}^{\sigma}, n_{r 2}^{\sigma}} \cdots x_{n_{r l_{r}}^{\sigma}, n_{r 1}^{\sigma}} \\
\operatorname{Mperm}_{n}(X) & =\sum_{\sigma \in S_{n}} x_{n_{11}^{\sigma}, n_{12}^{\sigma}} \cdots x_{n_{l_{r}}^{\sigma}, n_{11}^{\sigma}} \cdots x_{n_{r 1}^{\sigma}, n_{r 2}^{\sigma}} \cdots x_{n_{r l_{r}}^{\sigma}, n_{r 1}^{\sigma}}
\end{aligned}
$$

In the setting of a field $\mathbb{F}$ of characteristic 0, Alexander Barvinok, in $[\mathrm{B}$, has studied another variant of the noncommutative determinant called the symmetrized determinant, which is denoted $\operatorname{sdet}_{n}(X)$. It is defined as follows:

$$
\operatorname{sdet}_{n}(X)=\frac{1}{n !} \sum_{\sigma, \tau \in S_{n}} \operatorname{sgn}(\sigma) \operatorname{sgn}(\tau) x_{\tau(1), \sigma(1)} x_{\tau(2), \sigma(2)} \cdots x_{\tau(n), \sigma(n)} .
$$

Barvinok shows that, for any fixed dimensional associative algebra $\mathcal{A}$ over $\mathbb{F}$ of characteristic zero, there is a polynomial-time algorithm which, on input an $n \times n$ matrix $A$ with entries from $\mathcal{A}$,

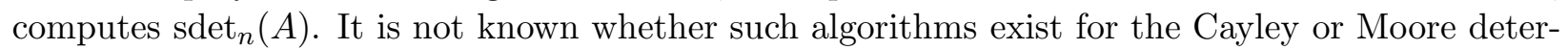
minants.

\subsection{Models for noncommutative arithmetic computation}

A noncommutative arithmetic circuit $C$ over a field $\mathbb{F}$ is defined as follows: $C$ is a directed acyclic graph and every leaf of the graph is labelled with either an input variable from the set of variables $X$ or an element from $\mathbb{F}$. Every internal node is labelled by either $(+)$ or $(\times)$ - meaning that it is either an addition or multiplication gate respectively - and has fanin two. Since we are working over noncommutative domains, we will assume that each multiplication gate has a designated left child and a designated right child. Each gate of the circuit computes a polynomial in $\mathbb{F}\langle X\rangle$ in the natural way: the polynomials computed at the leaves are the polynomials labelling the leaves; the polynomial computed at an internal node labelled by + (resp. $\times$ ) is the sum (resp. product in left-to-right order) of the polynomials computed at its children. The polynomial computed by $C$ is the polynomial computed at a designated output node of the circuit.

We also recall the definition of an Algebraic Branching Program (ABP) computing a noncommutative polynomial in $\mathbb{F}\langle X\rangle$ ([N91], RS05]). An ABP is a directed acyclic graph with one vertex of in-degree zero, which is called the source, and one vertex of out-degree zero, which is called the sink. The vertices of the graph are partitioned into levels numbered $0,1, \ldots, d$. Edges may only go from level $i$ to level $i+1$ for $i=0,1, \ldots, d-1$. The source is the only vertex at level 0 and the sink is the only vertex at level $d$. Each edge is labeled with a homogeneous linear form in the variables $X$. The size of the $\mathrm{ABP}$ is the number of vertices.

The ABP computes a degree $d$ homogeneous polynomial $f \in \mathbb{F}\langle X\rangle$ as follows. Fix any path $\gamma$ from source to sink with edges $e_{1}, e_{2}, \ldots, e_{d}$, where $e_{i}$ is the edge from level $i-1$ to level $i$, and let $\ell_{i}$ 
denote the linear form labelling edge $e_{i}$. We denote by $f_{\gamma}$ the homogeneous degree $d$ polynomial $\ell_{1} \cdot \ell_{2} \cdots \ell_{d}$ (note that the order of multiplication is important). The polynomial $f$ computed by the ABP is simply

$$
f=\sum_{\gamma \in \mathcal{P}} f_{\gamma}
$$

where $\mathcal{P}$ is the set of all paths from the source to the sink.

We will also consider a slight variant of the above definition where we allow multiple sources and sinks. A multi-output ABP $P$ is defined exactly as above, except that we allow multiple sources at level 0 and multiple sinks at level $d$. For each source $s$ and sink $t$, an ABP $P_{s t}$ may be obtained from $P$ by removing all sources other than $s$ and sinks other than $t$. Let $S=\left\{s_{1}, s_{2}, \ldots, s_{a}\right\}$ and $T=\left\{t_{1}, t_{2}, \ldots, t_{b}\right\}$ denote the sets of sources and sinks respectively in $P$. The ABP $P$ will be thought of as computing the $a b$ many polynomials computed by the ABPs $P_{s_{i}, t_{j}}$. More precisely, the output of the ABP $P$ is an $a \times b$ matrix $A$ with entries from $\mathbb{F}\langle X\rangle$ such that the $(i, j)$ th entry of $A$, denoted $A(i, j)$, is the polynomial computed by the $\mathrm{ABP} P_{s_{i}, t_{j}}$. It is easily seen that we can write $A$ as $\sum_{m \in \mathcal{M}_{d}(X)} A_{m} m$, where $A_{m} \in \mathbb{F}^{a \times b}$; we will call $A_{m}$ the coefficient matrix of the monomial $m$ in the matrix $A$.

\section{The Hadamard Product}

A key notion we require for all our reductions is the Hadamard product of polynomials that was introduced in AJS09.

Definition 1. Given polynomials $f, g \in \mathbb{F}\langle X\rangle$, their Hadamard product $h=f \circ g$ is defined as follows: $h$ is the unique polynomial in $\mathbb{F}\langle X\rangle$ such that for any monomial $m \in \mathcal{M}(X)$, the coefficient $h(m)=f(m) \cdot g(m)$.

In [AJS09, Theorem 5] we show that given a noncommutative circuit for polynomial $f$ and an ABP for polynomial $g$ we can efficiently compute a noncommutative circuit for their Hadamard product $f \circ g$. However, the construction we present in AJS09 modifies the noncommutative circuit for the polynomial $f$. Hence, it will not work if we are allowed only black-box access to $f$, which we require for certain applications in this paper.

Suppose we have an efficient black-box algorithm for evaluating the polynomial $f \in \mathbb{F}\langle X\rangle$, where the variables in $X$ take values in some matrix algebra (say, $n \times n$ matrices over a field $\mathbb{F}$ ). Furthermore, suppose we have an explicit ABP for the polynomial $g$. Ideally, we would like to obtain an efficient algorithm for computing their Hadamard product $f \circ g$ over the same matrix algebra.

However, what we can show is that we can put together the ABP and the black-box algorithm for $f$ to obtain an efficient algorithm that computes $f \circ g$ over $\mathbb{F}$. This turns out to be sufficient to prove all our hardness results for the different noncommutative determinants.

Theorem 2. Fix $d \in \mathbb{N}$. Let $Z=\left\{z_{1}, z_{2}, \ldots, z_{m}\right\}$ be a set of noncommuting variables and $g \in \mathbb{F}\langle Z\rangle$ be a homogeneous polynomial of degree $d$ such that $g$ is computed by an ABP P of size $S$. Then, there exist matrices $A_{1}, A_{2}, \ldots, A_{n} \in M_{S}(\mathbb{F})$ such that for any homogeneous polynomial $f \in \mathbb{F}\langle Z\rangle$ 
of degree $d, f \circ g=f\left(A_{1} z_{1}, A_{2} z_{2}, \ldots, A_{n} z_{n}\right)(1, S)$. Moreover, given the ABP P, the matrices $A_{1}, A_{2}, \ldots, A_{n}$ can be computed in time polynomial in the size of the description of $P$.

Proof. Let the vertices of $P$ be named $1,2, \ldots, S$ where 1 is the source of the ABP and $S$ is the sink. Define the matrices $A_{1}, A_{2}, \ldots, A_{n} \in M_{S}(\mathbb{F})$ as follows: $A_{i}(k, l)$ is the coefficient of the variable $z_{i}$ in the linear form labelling the edge that goes from vertex $k$ to vertex $l$; if there is no such edge, the entry $A_{i}(k, l)=0$. For any monomial $m=z_{i_{1}} z_{i_{2}} \cdots z_{i_{d}} \in \mathcal{M}_{d}(Z)$, let $A_{m}$ denote the matrix $A_{i_{1}} A_{i_{2}} \cdots A_{i_{d}}$. We see that

$$
\begin{aligned}
f\left(A_{1} z_{1}, A_{2} z_{2}, \ldots, A_{n} z_{n}\right) & =\sum_{i_{1}, i_{2}, \ldots, i_{d} \in[n]} f\left(z_{i_{1}} z_{i_{2}} \cdots z_{i_{d}}\right)\left(A_{i_{1}} z_{i_{1}}\right)\left(A_{i_{2}} z_{i_{2}}\right) \cdots\left(A_{i_{d}} z_{i_{d}}\right) \\
& =\sum_{i_{1}, i_{2}, \ldots, i_{d} \in[n]} f\left(z_{i_{1}} z_{i_{2}} \cdots z_{i_{d}}\right)\left(A_{i_{1}} A_{i_{2}} \cdots A_{i_{d}}\right)\left(z_{i_{1}} z_{i_{2}} \cdots z_{i_{d}}\right) \\
& =\sum_{m \in \mathcal{M}_{d}(Z)} f(m) A_{m} m
\end{aligned}
$$

Note that the coefficient $g(m)$ of a monomial $m=z_{i_{1}} z_{i_{2}} \cdots z_{i_{d}}$ in $g$ is just $A_{m}(1, S)=$ $\sum_{k_{1}, k_{2}, \ldots, k_{d-1} \in[S]} \prod_{j=1}^{d} A_{i_{j}}\left(k_{j-1}, k_{j}\right)$, where $k_{0}=1$ and $k_{d}=S$. Putting the above observations together, we see that $f\left(A_{1} z_{1}, A_{2} z_{2}, \ldots, A_{n} z_{n}\right)(1, S)=\sum_{m \in \mathcal{M}_{d}(Z)} f(m) A_{m}(1, S) m=$ $\sum_{m \in \mathcal{M}_{d}(Z)} f(m) g(m) m=f \circ g$. Since the entries of the matrices $A_{1}, A_{2}, \ldots, A_{n}$ can be read off from the labels of $P$, it can be seen that $A_{1}, A_{2}, \ldots, A_{n}$ can be computed in polynomial time given the $\mathrm{ABP} P$. This completes the proof.

Remark 3. We note that the matrices $A_{i}$ in the statement of Theorem $Q$ can actually be computed from the $A B P$ even more efficiently, say, in uniform $\mathrm{AC}^{0}$.

The following corollary is immediate.

Corollary 4. AJS09] Given a noncommutative circuit of size $S^{\prime}$ for $f \in \mathbb{F}\langle Z\rangle$ and an $A B P$ of size $S$ for $g \in \mathbb{F}\langle Z\rangle$, we can efficiently compute a noncommutative circuit of size $O\left(S^{\prime} S^{3}\right)$ for $f \circ g$.

The next corollary is the more useful version for this paper.

Corollary 5. Let $Z=\left\{z_{1}, z_{2}, \ldots, z_{n}\right\}$. Suppose $\mathcal{A}$ is a polynomial-time algorithm for computing a homogeneous degree $d$ polynomial $f \in \mathbb{F}\langle Z\rangle$ for matrix inputs from $M_{S}(\mathbb{F}) 2$ Given as input an $A B P P$, with $S$ nodes, computing a homogeneous degree d polynomial $g \in \mathbb{F}\langle Z\rangle$, and scalars $a_{1}, a_{2}, \ldots, a_{n} \in \mathbb{F}$, we can compute $f \circ g\left(a_{1}, a_{2}, \ldots, a_{n}\right)$ in polynomial time.

Proof. We first compute matrices $A_{1}, A_{2}, \ldots, A_{n}$, described in the Theorem 2 , in time polynomial in the description of the ABP $P$. Then we invoke the given algorithm $\mathcal{A}$ on input $\left(A_{1} a_{1}, A_{2} a_{2}, \ldots, A_{n} a_{n}\right)$ to obtain as output an $S \times S$ matrix whose $(1, S)^{\text {th }}$ entry contains $f \circ g\left(a_{1}, a_{2}, \ldots, a_{n}\right)$. Clearly, the simulation runs in polynomial time.

\footnotetext{
${ }^{2}$ The statement can be generalized to any unital algebra $\mathcal{A}$ in place of the field $\mathbb{F}$.
} 


\section{The hardness of the Cayley determinant}

We consider polynomials over an arbitrary field $\mathbb{F}$ (for the algorithmic results $\mathbb{F}$ is either rational numbers or a finite field). The main result of this section is that if there is a polynomial-time algorithm to compute the $2 n \times 2 n$ Cayley determinant over inputs from $M_{S}\left(\mathbb{F}\right.$ ) for $S=c \cdot n^{2}$ (for a suitable constant $c$ ) then there is a polynomial-time algorithm to compute the $n \times n$ permanent over $\mathbb{F}$.

Throughout this section let $X$ denote $\left\{x_{i j} \mid 1 \leq i, j \leq 2 n\right\}$, and $Y$ denote $\left\{y_{i j} \mid 1 \leq i, j \leq n\right\}$. Our aim is to show that if there is a polynomial-time algorithm for computing $\operatorname{Cdet}_{2 n}(X)$ where $x_{i j}$ takes values in $M_{S}(\mathbb{F})$ then there is a polynomial-time algorithm that computes $\operatorname{Cperm}_{n}(Y)$ where $y_{i j}$ takes values in $\mathbb{F}$.

The $2 n \times 2 n$ determinant has $2 n$ ! many signed monomials of degree $2 n$ of the form $x_{1, \sigma(1)} x_{2, \sigma(2)} \cdots x_{2 n, \sigma(2 n)}$ for $\sigma \in S_{2 n}$. We will identify $n$ ! of these monomials, all of which have the same sign. More precisely, we will design a small ABP with which we will be able to pick out these $n$ ! monomials of the same sign.

We now define these $n$ ! many permutations from $S_{2 n}$ which have the same sign and the corresponding monomials of $\operatorname{Cdet}_{2 n}$ that can be picked out by a small ABP.

Definition 6. Let $n \in \mathbb{N}$. For each permutation $\pi \in S_{n}$, we define a permutation $\rho(\pi)$ in $S_{2 n}$, called the interleaving of $\pi$, as follows:

$$
\rho(\pi)(i)= \begin{cases}\pi\left(\frac{i+1}{2}\right), & \text { if } i \text { is odd }, \\ n+\pi\left(\frac{i}{2}\right), & \text { if } i \text { is even } .\end{cases}
$$

That is, the elements $\rho(\pi)(1), \rho(\pi)(2), \cdots, \rho(\pi)(2 n)$ are simply $\pi(1),(n+\pi(1)), \pi(2),(n+$ $\pi(2)), \cdots, \pi(n),(n+\pi(n))$.

The following lemma states a crucial property of the permutation $\rho(\pi)$.

Lemma 7. The sign of the permutation $\rho(\pi)$ is independent of $\pi$. More precisely, for every $\pi \in S_{n}$, we have $\operatorname{sgn}(\rho(\pi))=\operatorname{sgn}\left(\rho\left(1_{n}\right)\right)$, where $1_{n}$ denotes the identity permutation in $S_{n}$.

Proof. For each $\pi \in S_{n}$ we can define the permutation $\pi_{2} \in S_{2 n}$ as $\pi_{2}(i)=\pi(i)$ for $1 \leq i \leq n$ and $\pi_{2}(n+j)=n+\pi(j)$ for $1 \leq j \leq n$. It is easy to verify that $\operatorname{sgn}\left(\pi_{2}\right)=\operatorname{sgn}(\pi)^{2}=1$ for every $\pi \in S_{n}$. To see this we write $\pi_{2}$ as a product of disjoint cycles and notice that every cycle occurs an even number of times. Furthermore, we can check that $\rho(\pi)=\rho\left(1_{n}\right) \pi_{2}$, where we evaluate products of permutations from left to right. Hence it follows that $\operatorname{sgn}(\rho(\pi))=\operatorname{sgn}\left(\rho\left(1_{n}\right)\right) \operatorname{sgn}\left(\pi_{2}\right)=$ $\operatorname{sgn}\left(\rho\left(1_{n}\right)\right)$.

We will denote by $\rho_{0}$ the permutation $\rho\left(1_{n}\right)$, where $1_{n}$ denotes the identity permutation in $S_{n}$.

For $\sigma \in S_{2 n}$, we will denote by $m_{\sigma}$ the monomial $x_{1, \sigma(1)} x_{2, \sigma(2)} \cdots x_{2 n, \sigma(2 n)} \in \mathcal{M}(X)$. For $\sigma, \tau \in S_{2 n}$, we will denote the monomial $x_{\sigma(1), \tau(1)} x_{\sigma(2), \tau(2)} \cdots x_{\sigma(2 n), \tau(2 n)}$ by $m_{\sigma, \tau}$. 
In the next lemma we show that there is an ABP that will filter out monomials that are not of the form $m_{\rho(\pi)}$ from among the $m_{\sigma}$.

Lemma 8. There is an $A B P P$ of size $O\left(n^{2}\right)$ and width $n$ that computes a homogeneous polynomial $F \in \mathbb{F}\langle X\rangle$ of degree $2 n$ such that for any $\sigma, \tau \in S_{2 n}$,

- $F\left(m_{\sigma}\right)=1$ if $\sigma=\rho(\pi)$ for some $\pi \in S_{n}$, and 0 otherwise.

- $F\left(m_{\sigma, \tau}\right)=0$ unless $\sigma=1_{2 n}$, where $1_{2 n}$ denotes the identity permutation in $S_{2 n}$.

Moreover, the above ABP P can be computed in time $\operatorname{poly}(n)$.

Proof. The ABP is essentially just a finite automaton over the alphabet $X$ with the following properties: for input monomials of the form $m_{\sigma}$ it accepts only those monomials that are of the form $m_{\rho(\pi)}$. Further, for input monomials of the form $m_{\sigma, \tau}$ it accepts only those monomials of the form $m_{1_{2 n}, \tau}$. We give the formal description of this ABP $P$ below.

The ABP $P$ contains $2 n+1$ layers, labelled $\{0,1, \ldots, 2 n\}$. For each even $i \in\{0,1, \ldots, 2 n\}$, there is exactly one node $q_{i}$ at level $i$; for each odd $i \in\{0,1, \ldots, 2 n\}$, there are $n$ nodes $p_{i, 1}, p_{i, 2}, \ldots, p_{i, n}$ at level $i$. We now describe the edges of $P$ : for each even $i \in\{0,1, \ldots, 2 n-2\}$ and $j \in[n]$, there is an edge from $q_{i}$ to $p_{i+1, j}$ labelled $x_{i+1, j}$; for each odd $i \in\{0,1, \ldots, 2 n\}$ and $j \in[n]$, there is an edge from $p_{i, j}$ to $q_{i+1}$ labelled $x_{i+1, n+j}$.

It is easy to see that $P$ as defined above satisfies the requirements of the statement of the lemma. It is also clear that the ABP $P$ can be computed in polynomial time.

Note that the ABP $P$ of Lemma 8 can in fact be constructed in uniform $\mathrm{AC}^{0}$.

Remark 9. For this section we require only the first part of Lemma 8 . The second part of Lemma 8 is used in Section 6 .

We are now ready to prove that if there is a small noncommutative arithmetic circuit that computes the Cayley determinant polynomial, then there is a small noncommutative arithmetic circuit that computes the Cayley permanent polynomial.

Theorem 10. For any $n \in \mathbb{N}$, if there is a circuit $C$ of size $s$ computing $\operatorname{Cdet}_{2 n}(X)$, then there is a circuit $C^{\prime}$ of size polynomial in $s$ and $n$ that computes $\operatorname{Cperm}_{n}(Y)$.

Proof. Assuming the existence of the circuit $C$ as stated above, by Corollary 4 , there is a noncommutative arithmetic circuit $C^{\prime \prime}$ of size poly $(s, n)$ that computes the polynomial $F^{\prime \prime}=\operatorname{Cdet}_{2 n} \circ F$, where $F$ is the polynomial referred to in Lemma 8. For any monomial $m$, if $m \neq m_{\sigma}$ for any $\sigma \in S_{2 n}$, then $\operatorname{Cdet}_{2 n}(m)=0$ and hence, in this case, $F^{\prime \prime}(m)=0$; moreover, for $m=m_{\sigma}$, we have $F(m)=1$ if $\sigma=\rho(\pi)$ for some $\pi \in S_{n}$, and 0 otherwise. Hence, we see that

$$
F^{\prime \prime}(X)=\sum_{\pi \in S_{n}} \operatorname{sgn}(\rho(\pi)) m_{\rho(\pi)}=\operatorname{sgn}\left(\rho_{0}\right)\left(\sum_{\pi \in S_{n}} m_{\rho(\pi)}\right)
$$


where the last equality follows from Lemma 7.

Let $C^{\prime}$ be the circuit obtained from $C^{\prime \prime}$ by substituting $x_{i j}$ with $y_{\frac{1+i}{2}, j}$ if $i$ is odd and $j \in[n]$, and by 1 if $i$ is even or $j \notin[n]$, and by multiplying the output of the resulting circuit by $\operatorname{sgn}\left(\rho_{0}\right)$. Let $F^{\prime}$ denote the polynomial computed by $C^{\prime}$. Then, we have

$$
F^{\prime}(X)=\sum_{\pi \in S_{n}} m_{\rho(\pi)}^{\prime}
$$

where $m_{\rho(\pi)}^{\prime}$ denotes the monomial obtained from $m_{\rho(\pi)}$ after the substitution. It can be checked that for any $\pi \in S_{n}$, the monomial $m_{\rho(\pi)}^{\prime}=y_{1, \pi(1)} y_{2, \pi(2)} \cdots y_{n, \pi(n)}$. Hence, the polynomial $F^{\prime}$ computed by $C^{\prime}$ in indeed $\operatorname{Cperm}_{n}(Y)$. It is easily seen that the size of $C^{\prime}$ is $\operatorname{poly}(s, n)$.

We now show that evaluating the polynomial $\operatorname{Cdet}_{2 n}$ over $M_{S}(\mathbb{F})$, for $S=c \cdot n^{2}$ for suitable $c>0$, is at least as hard as evaluating the permanent over $\mathbb{F}$.

Theorem 11. If there is a polynomial-time algorithm $\mathcal{A}$ that computes the $2 n \times 2 n$ Cayley determinant of matrices with entries in $M_{S}(\mathbb{F})$, for $S=c \cdot n^{2}$ for suitable $c>0$, then there is a polynomial-time algorithm that computes the $n \times n$ permanent over $\mathbb{F}$.

Proof. This is an easy consequence of Corollary 5. Consider the algorithm given by Corollary 5 for computing $\operatorname{Cdet}_{2 n} \circ F$ over the field $\mathbb{F}$, where the ABP in Corollary 5 is the ABP of Lemma 8 computing $F$.

In order to evaluate the permanent over inputs $a_{i j}, 1 \leq i, j \leq n$ we will substitute $x_{2 i-1, j}=a_{i j}$ for $1 \leq i, j \leq n$ and we put $x_{i, j}=1$ when $i$ is even or $j>n$. As in the proof of Theorem 10 it follows that for this substitution the algorithm computing $\operatorname{Cdet}_{2 n} \circ F$ will output $\operatorname{sgn}\left(\rho_{0}\right) \operatorname{Cperm}_{n}\left(a_{11}, \ldots, a_{n n}\right)$. Since $\operatorname{sgn}\left(\rho_{0}\right)$ can be easily computed, we have a polynomial-time algorithm for computing the $n \times n$ permanent over $\mathbb{F}$.

Remark 12. The above result has a stronger consequence: for any fixed $\varepsilon>0$, if there is a polynomial-time algorithm that computes the $m \times m$ Cayley determinant over $M_{m^{\varepsilon}}(\mathbb{F})$, then there is a polynomial-time algorithm that computes $\Omega\left(m^{\varepsilon / 2}\right) \times \Omega\left(m^{\varepsilon / 2}\right)$ permanents over $\mathbb{F}$, hence implying that permanent over $\mathbb{F}$ is polynomial-time computable.

\section{The Cayley determinant over Clifford algebras}

We now consider the complexity of computing the determinant over real Clifford algebras of polynomially large dimension. We show via a polynomial-time reduction that computing the permanent over rationals is reducible to this problem. Indeed, by inspecting our result we can observe that even approximating the determinant over such Clifford algebras would yield similar approximation algorithms for the permanent over the reals.

We first define the basic notions in the theory of Clifford algebras. A thorough treatment can be found in [LS09]. Fix $m \in \mathbb{N}$. The (real) Clifford algebra $C L_{m}^{\prime}$ is a $2^{m}$-dimensional vector space 
over $\mathbb{R}$ with basis elements of the form $e_{i_{1}} e_{i_{2}} e_{i_{3}} \cdots e_{i_{k}}$ where $i_{1}<i_{2}<i_{3} \cdots<i_{k}$ are elements from $[m]$. Multiplication between elements of the basis is defined by the following rules: $e_{i}^{2}=1$ and $e_{i} e_{j}=-e_{j} e_{i}$ for distinct $i, j \in[m]$; this is extended linearly to all pairs of elements from the Clifford algebra. Given $i_{1}<i_{2}<\cdots<i_{k}$ from $[m]$, we denote by $e_{S}$ the basis element $e_{i_{1}} e_{i_{2}} \cdots e_{i_{k}}$, where $S=\left\{i_{1}, i_{2}, \ldots, i_{k}\right\}$. Each element of the Clifford algebra is uniquely expressible as $\sum_{S \subseteq[m]} c_{S} e_{S}$, where $c_{S} \in \mathbb{R}$ for each $S$. (Note that $e_{\emptyset}$ and 1 both refer to the multiplicative identity of the algebra.) An idempotent of the Clifford algebra is an element $e$ such that $e^{2}=e$. Given $h=\sum_{S \subseteq[m]} c_{S} e_{S}$ in $C L_{m}^{\prime}$, we define its norm $|h|$ to be $\sqrt{\sum_{S \subseteq[m]} c_{S}^{2}}$.

The subset of basis elements $\left\{e_{S} \mid S\right.$ has even cardinality $\}$ generates a strict subalgebra of $C L_{m}^{\prime}$. We will denote this subalgebra by $C L_{m}$. This is the algebra of interest to us. The term 'Clifford algebra' will henceforth refer to $C L_{m}$ for some $m \in \mathbb{N}$.

Chien, Rasmussen, and Sinclair [CRS03 have shown that a polynomial-time algorithm that, when given as input an $n \times n$ matrix $B$ with entries from $C L_{m}$ for $m=2 \log n+2$, $\operatorname{computes}\left|\operatorname{Cdet}_{n}(B)\right|^{2}$ can be used to design a randomized polynomial time algorithm to approximate the 0-1 permanent (over $\mathbb{Q})$.

In this section, we prove that if there is a polynomial-time algorithm to compute either $\left|\operatorname{Cdet}_{n}(B)\right|^{2}$ or $\operatorname{Cdet}_{n}(B)$, then the permanent (over inputs from $\mathbb{R}$ ) can actually be computed in polynomial time. For an $n \times n$ real matrix $A$, let $\operatorname{perm}_{n}(A)$ denote the permanent of $A$.

Remark 13. In a sense, our result in this section should not be surprising. We have already proved (in Theorem [11) that computing the determinant over matrix algebras is at least as hard as computing the permanent. Also, it is known that Clifford algebras of polynomial dimension are isomorphic to matrix algebras of polynomial dimension (see, for example, [LS09, Chapter 5]). However, in this section we actually give an explicit polynomial-time reduction showing that computing the permanent over the reals is reducible to computing either $\left|\operatorname{Cdet}_{n}(B)\right|^{2} \operatorname{or}_{\operatorname{Cdet}_{n}(B) \text { where the }}$ entries of $B$ are from the Clifford algebra $C L_{m}$.

Suppose we wish to compute the permanent of an $n \times n$ matrix with entries from $\mathbb{R}$. W.l.o.g., we assume that $n=2^{\ell}$ for some $\ell \in \mathbb{N}$. Let $m$ denote $5 \ell$. The next lemma is about the existence of certain elements in the algebra $C L_{m}$ useful for the reduction.

Lemma 14. Let $n, \ell, m$ be as above. Then, there exist $h_{1}, h_{2}, \ldots, h_{n}, h_{1}^{\prime} h_{2}^{\prime}, \ldots, h_{n}^{\prime} \in C L_{m}$ and an idempotent $e \in C L_{m}$ such that:

- For all $j, h_{j} h_{j}^{\prime}=e$.

- For all $j \neq k, h_{j} h_{k}^{\prime}=0$.

- $|e|^{2}=\frac{1}{2^{\ell}}$.

Moreover, the elements $h_{1}, h_{2}, \ldots, h_{n}, h_{1}^{\prime}, h_{2}^{\prime}, \ldots, h_{n}^{\prime}$ and e can be constructed in time poly $(n)$.

We defer the proof of the above lemma and first prove the main result of this section. 
Theorem 15. Let $n, \ell, m$ be as above. There is a polynomial-time algorithm which, when given any matrix $A \in M_{n}(\mathbb{R})$, computes a $B \in M_{2 n}\left(C L_{m}\right)$ such that $\left|\operatorname{Cdet}_{2 n}(B)\right|^{2}=\frac{\operatorname{perm}_{n}(A)^{2}}{2^{\ell}}$.

Proof. The matrix $B$ will be the following: for any odd $i \in[2 n]$ and any $j \in[2 n]$, set $B(i, j)-$ the $(i, j)$ th entry of $B$ - to be $A\left(\frac{i+1}{2}, j\right) h_{j}$ if $j \leq n$ and 0 if $j>n$; for any even $i \in[2 n]$ and $j \in[2 n]$, set $B(i, j)$ to be $h_{j-n}^{\prime}$ if $j>n$ and 0 otherwise. Clearly, $B$ can be computed in polynomial time given $A$. Note the following property of $B$ : for any odd $i \in[2 n]$ and $j, k \in[2 n]$

$$
B(i, j) B(i+1, k)=\left\{\begin{array}{lr}
A\left(\frac{i+1}{2}, j\right) e & \text { if } j \leq n \text { and } k=n+j \\
0 & \text { otherwise }
\end{array}\right.
$$

Here $e$ denotes the idempotent from Lemma 14, The following claim is easy to see.

Claim 16. For any permutation $\sigma \in S_{2 n}$, the product $\prod_{i=1}^{2 n} B(i, \sigma(i))=\left(\prod_{i=1}^{n} A(i, \pi(i))\right)$ e if $\sigma=\rho(\pi)$ for some $\pi \in S_{n}$ and it is 0 otherwise.

Let us consider $\operatorname{Cdet}_{2 n}(B)$. We have:

$$
\begin{aligned}
\operatorname{Cdet}_{2 n}(B) & =\sum_{\sigma \in S_{2 n}} \operatorname{sgn}(\sigma) B(1, \sigma(1)) \cdot B(2, \sigma(2)) \cdots B(2 n, \sigma(2 n)) \\
& =\sum_{\pi \in S_{n}} \operatorname{sgn}(\rho(\pi))\left(\prod_{i=1}^{n} A(i, \pi(i))\right) e \\
& =\operatorname{sgn}\left(\rho_{0}\right) \operatorname{perm}_{n}(A) e
\end{aligned}
$$

Thus, we see that $\left|\operatorname{Cdet}_{2 n}(B)\right|^{2}=\operatorname{perm}_{n}(A)^{2}|e|^{2}=\frac{\operatorname{perm}_{n}(A)^{2}}{2^{\ell}}$.

We have the following easy consequence of the above theorem.

Corollary 17. Fix any $\varepsilon>0$, and suppose there is a polynomial-time algorithm that computes $\left|\operatorname{Cdet}_{n}(B)\right|^{2}$ on input an $n \times n$ matrix $B$ with entries from $C L_{m}$ for $m=\varepsilon \log n$. Then there is a polynomial-time algorithm that computes the $n \times n$ permanent of matrices with nonnegative rational entries.

Proof. The statement directly follows from Theorem 15 for $m=\lceil 5 \log n\rceil$. To prove hardness for $m=\varepsilon \log n$, we note that a polynomial-time algorithm to $\operatorname{compute}\left|\operatorname{Cdet}_{n}(B)\right|^{2}$ over $C L_{\varepsilon} \log n$ can be used to compute $\left|\operatorname{Cdet}_{n^{\varepsilon / 5}}(B)\right|^{2}$ over $C L_{5 \log n^{\varepsilon / 5}}$ in polynomial time.

A $\delta$-approximation algorithm $\mathcal{A}$ for a function $f: \Sigma^{*} \longrightarrow \mathbb{Q}$ is an algorithm such that for each $x \in \Sigma^{*}$

$$
(1-\delta) f(x) \leq \mathcal{A}(x) \leq(1+\delta) f(x)
$$

Our reduction from computing the permanent for nonnegative entries to computing $\left|\operatorname{Cdet}_{n}(B)\right|^{2}$ actually yields an approximation preserving reduction. We formalize this in the next corollary. 
Corollary 18. Fix any $\delta>0$ and $\varepsilon>0$. Suppose there is a polynomial-time $\delta$-approximation algorithm for the function that on input an $n \times n$ matrix $B$ with entries from $C L_{m}$ for $m=\varepsilon \log n$ takes the value $\left|\operatorname{Cdet}_{n}(B)\right|^{2}$. Then there is a polynomial-time $\delta$-approximation algorithm for the $n \times n$ permanent with nonnegative rational entries.

We now prove Lemma 14

Proof of Lemma 14. Let $e_{1}, e_{2}, \ldots, e_{m}$ denote the generators of $C L_{m}^{\prime}$. Partition the set $[m]$ into $\ell$ subsets of size 5 as follows: set $S_{i}=\{5(i-1)+j \mid j \in[5]\}$ for each $i \in[\ell]$. For each $i \in[\ell]$, let $S_{i, 0}=\{5(i-1)+1,5(i-1)+2,5(i-1)+3,5(i-1)+5\}$ and $S_{i, 1}=\{5(i-1)+2,5(i-1)+3,5(i-$ 1) $+4,5(i-1)+5\}$.

Using the fact that $e_{i}^{2}=1$ and $e_{i} e_{j}=-e_{j} e_{i}$ for $i \neq j$ it easily follows that for any two disjoint sets $S, T \subseteq[m]$ such that $|S|,|T|$ are even, we have $e_{S} e_{T}=e_{T} e_{S}$. Hence, the elements $e_{S_{i, b_{1}}}$ and $e_{S_{j, b_{2}}}$ commute for $i \neq j$ and any $b_{1}, b_{2} \in\{0,1\}$. Furthermore, for all $i \in[\ell]$ and $b \in\{0,1\}$ we have $e_{S_{i, b}}^{2}=1$. Also, we have $e_{S_{i, 0}} e_{S_{i, 1}}=-e_{S_{i, 1}} e_{S_{i, 0}}$. Finally, notice that $e_{S_{i, b}}$ for $1 \leq i \leq \ell$ and $b \in\{0,1\}$ are all elements of $C L_{m}$.

For $i \in[\ell]$ and $b \in\{0,1\}$, set $g_{i, 0}=\frac{1+e_{S_{i, 1}}}{2}$ and $g_{i, 1}=\frac{e_{S_{i, 0}}\left(1-e_{S_{i, 1}}\right)}{2}$. Also, set $g_{i, 0}^{\prime}=g_{i, 0}$ and $g_{i, 1}^{\prime}=\frac{e_{S_{i, 0}}\left(1+e_{S_{i, 1}}\right)}{2}$. Notice that $g_{i, 0}^{2}=g_{i, 0}$. We also note an additional relation $e_{S_{i, 0}}\left(1-e_{S_{i, 1}}\right)=$ $\left(1+e_{S_{i, 1}}\right) e_{S_{i, 0}}$. Using these we can easily derive the following crucial properties of these elements of $C L_{m}$.

- For each $i \in[\ell]$ and $b \in\{0,1\}, g_{i, b} g_{i, b}^{\prime}=g_{i, 0}$.

- For each $i \in[\ell]$ and $b \in\{0,1\}, g_{i, b} g_{i, 1-b}^{\prime}=0$.

- For $i_{1} \neq i_{2}$ and any $b_{1}, b_{2} \in\{0,1\}$, the elements $g_{i_{1}, b_{1}}$ and $g_{i_{2}, b_{2}}^{\prime}$ commute.

Finally, we define $h_{j}, h_{j}^{\prime}$ for a fixed $j \in[n]$. Let $b_{1} b_{2} \ldots b_{\ell}$ be the binary representation of the integer $j-1$ (recall that $n=2^{\ell}$ ). We define $h_{j}=g_{1, b_{1}} g_{2, b_{2}} \cdots g_{\ell, b_{\ell}}$ and $h_{j}^{\prime}=g_{1, b_{1}}^{\prime} g_{2, b_{2}}^{\prime} \cdots g_{\ell, b_{\ell}}^{\prime}$. Also, we define $e$ to be $g_{1,0} g_{2,0} \cdots g_{\ell, 0}$, which is the same as $h_{1}$ and $h_{1}^{\prime}$.

We now prove that the $h_{j}, h_{j}^{\prime}(j \in[n])$ and $e$ satisfy the properties claimed in the statement of the lemma. Fix any $j \in[n]$ and let $b_{1} b_{2} \ldots b_{\ell}$ be the binary representation of $j-1$. We have

$$
\begin{aligned}
h_{j} h_{j}^{\prime} & =g_{1, b_{1}} g_{2, b_{2}} \cdots g_{\ell, b_{\ell}} g_{1, b_{1}}^{\prime} g_{2, b_{2}}^{\prime} \cdots g_{\ell, b_{\ell}}^{\prime} \\
& =\left(g_{1, b_{1}} g_{1, b_{1}}^{\prime}\right) \cdot\left(g_{2, b_{1}} g_{2, b_{2}}^{\prime}\right) \cdots\left(g_{\ell, b_{\ell}} g_{\ell, b_{\ell}}^{\prime}\right) \\
& =g_{1,0} g_{2,0} \cdots g_{\ell, 0}=e
\end{aligned}
$$

The second equality follows from the fact that $g_{i_{1}, b}$ and $g_{i_{2}, b}^{\prime}$ commute for any distinct $i_{1}$ and $i_{2}$. The third equality follows from the fact that for any $i$ and $b, g_{i, b} g_{i, b}^{\prime}=g_{i, 0}$. This proves the first property claimed in the statement of the lemma. Similarly, we can see that $e$ is an idempotent: $e^{2}=h_{1}^{2}=e$. 
Fix any distinct $j, k \in[n]$. Let $b_{1} b_{2} \ldots b_{\ell}$ and $b_{1}^{\prime} b_{2}^{\prime} \ldots b_{\ell}^{\prime}$ be the binary representations of $j$ and $k$. Since $j \neq k$, we can fix some $i$ such that $b_{i} \neq b_{i}^{\prime}$. We have

$$
\begin{aligned}
h_{j} h_{k}^{\prime} & =g_{1, b_{1}} g_{2, b_{2}} \cdots g_{\ell, b_{\ell}} g_{1, b_{1}^{\prime}}^{\prime} g_{2, b_{2}^{\prime}}^{\prime} \cdots g_{\ell, b_{\ell}^{\prime}}^{\prime} \\
& =\left(g_{1, b_{1}} g_{1, b_{1}}^{\prime}\right) \cdot\left(g_{2, b_{1}} g_{2, b_{2}}^{\prime}\right) \cdots\left(g_{i, b_{i}} g_{i, b_{i}^{\prime}}^{\prime}\right) \cdots\left(g_{\ell, b_{\ell}} g_{\ell, b_{\ell}}^{\prime}\right) \\
& =\left(g_{1, b_{1}} g_{1, b_{1}}^{\prime}\right) \cdot\left(g_{2, b_{1}} g_{2, b_{2}}^{\prime}\right) \cdots 0 \cdots\left(g_{\ell, b_{\ell}} g_{\ell, b_{\ell}}^{\prime}\right)=0
\end{aligned}
$$

where the third equality follows from the fact that we have $g_{i, b} g_{i, 1-b}^{\prime}=0$. This proves the second claim made in the lemma.

Finally, we note that

$$
\begin{aligned}
|e|^{2} & =\left|g_{1,0} g_{2,0} \cdots g_{\ell, 0}\right|^{2}=\left|\frac{1}{2^{\ell}} \sum_{T \subseteq \ell} \prod_{i \in T} e_{S_{i, 1}}\right|^{2} \\
& =\frac{1}{4^{\ell}}\left|\sum_{T \subseteq \ell} \prod_{i \in T} e_{S_{i, 1}}\right|^{2}=\frac{2^{\ell}}{4^{\ell}}=\frac{1}{2^{\ell}}
\end{aligned}
$$

It is easily seen from their definitions that the $h_{j}, h_{j}^{\prime}$ and $e$ can be computed in time poly $(n)$. This completes the proof of the lemma.

\section{The Symmetrized Determinant}

In this section, we observe that the $2 n \times 2 n$ symmetrized determinant over $O\left(n^{2}\right)$-dimensional matrix algebras is at least as hard to compute as the permanent. This stands in marked contrast to the result of Barvinok $[\mathrm{B}]$, who shows that over constant-dimensional matrix algebras, the symmetrized determinant is polynomial-time computable.

In this section, let $\mathbb{F}$ denote a field of characteristic 0 . Let $X=\left\{x_{i j} \mid 1 \leq i, j \leq 2 n\right\}$ and $Y=$ $\left\{y_{i j} \mid 1 \leq i, j \leq n\right\}$. Recall that for $\sigma, \tau \in S_{2 n}$, the monomial $m_{\sigma, \tau}$ is $x_{\sigma(1), \tau(1)} x_{\sigma(2), \tau(2)} \cdots x_{\sigma(2 n), \tau(2 n)}$, and the monomial $m_{\sigma}$ is $x_{1, \sigma(1)} x_{2, \sigma(2)} \cdots x_{2 n, \sigma(2 n)}$.

Theorem 19. If the $\operatorname{sdet}_{2 n}(X)$ polynomial over $\mathbb{F}$ can be computed by a polynomial-sized noncommutative arithmetic circuit, then the polynomial $\mathrm{Cperm}_{n}(Y)$ can also be computed by a polynomialsized noncommutative arithmetic circuit.

Proof. Assume that $\operatorname{sdet}_{2 n}(X)$ is computed by a circuit $C$ of size $s$. As in Theorem 10, we will proceed by taking Hadamard product. Let $P$ be the ABP defined in Lemma 8 and $F(X)$ the polynomial it computes. Let $F^{\prime \prime}$ denote the polynomial $\operatorname{sdet}_{2 n}(X) \circ F$. Note that by Corollary 4 , $F^{\prime \prime}$ can be computed by a circuit $C^{\prime \prime}$ of size $\operatorname{poly}(s, n)$. From Lemma 8 , we have $F\left(m_{\sigma, \tau}\right)=0$ unless $\sigma=1_{2 n}$, the identity permutation in $S_{2 n}$; moreover, we also have $F\left(m_{1_{2 n}, \tau}\right)=F\left(m_{\tau}\right)$ which is 1 if $\tau=\rho(\pi)$ for some $\pi \in S_{n}$ and 0 otherwise. By the above reasoning,

$$
F^{\prime \prime}(X)=\frac{1}{(2 n) !} \sum_{\pi \in S_{n}} \operatorname{sgn}(\rho(\pi)) m_{\rho(\pi)}=\frac{\operatorname{sgn}\left(\rho_{0}\right)}{(2 n) !} \sum_{\pi \in S_{n}} m_{\rho(\pi)}
$$


Now, we substitute each $x_{i j}$ by $y_{\frac{1+i}{2}, j}$ if $i$ is odd and $j \in[n]$ and by 1 if $i$ is even or $j \notin[n]$ in the circuit $C^{\prime \prime}$. The effect of this substitution is to transform $m_{\rho(\pi)}$ into $y_{1, \pi(1)} y_{2, \pi(2)} \cdots y_{n, \pi(n)}$ for each $\pi \in S_{n}$. Hence, the resulting polynomial is simply $\frac{\operatorname{sgn}\left(\rho_{0}\right) \operatorname{Cperm}_{n}(Y)}{(2 n) !}$. Thus, by multiplying by $\operatorname{sgn}\left(\rho_{0}\right)(2 n)$ !, we obtain a circuit $C^{\prime}$ of size poly $(s, n)$ that computes $\operatorname{Cperm}_{n}(Y)$.

Theorem 20. If there is a polynomial-time algorithm $\mathcal{A}$ that computes the $2 n \times 2 n$ symmetrized determinant of matrices with entries in $M_{S}(\mathbb{F})$, for $S=c \cdot n^{2}$ for suitable $c>0$, then there is a polynomial-time algorithm that computes the $n \times n$ permanent over $\mathbb{F}$.

Proof. The proof is almost exactly identical to that of Theorem 11, Consider the algorithm given by Corollary 5 for computing $\operatorname{sdet}_{2 n} \circ F$ over the field $\mathbb{F}$, where the ABP in Corollary 5 is the ABP of Lemma 8 computing $F$.

In order to evaluate the permanent over inputs $a_{i j}, 1 \leq i, j \leq n$ we will substitute $x_{2 i-1, j}=a_{i j}$ for $1 \leq i, j \leq n$ and we put $x_{i, j}=1$ when $i$ is even or $j>n$. As in the proof of Theorem 19, it follows that for this substitution the algorithm computing $\operatorname{sdet}_{2 n} \circ F$ will output $\frac{\operatorname{sgn}\left(\rho_{0}\right)}{(2 n) !} \operatorname{Cperm}_{n}\left(a_{11}, \ldots, a_{n n}\right)$. Since $\operatorname{sgn}\left(\rho_{0}\right)$ and $(2 n) !$ are easily computable, we have a polynomialtime algorithm for computing the $n \times n$ permanent over $\mathbb{F}$.

\section{The Moore determinant}

We demonstrate by a simple reduction that the Moore determinant and permanent are interreducible. We also show that the computing the Moore determinant over a field of characteristic zero is at least as hard as counting the number of directed Hamilton Cycles of a directed graph, which is a well-known \# $\mathrm{P}$-complete problem. If the field is of characteristic $k$, then computing the Moore determinant over the field is at least as hard as counting the number of Hamilton cycles of a directed graph modulo the prime $k$, which is hard for $\operatorname{Mod}_{k} \mathrm{P}$.

Assume $X=\left\{x_{i j} \mid 1 \leq i, j \leq n\right\}$. Given a permutation $\sigma \in S_{n}$, we write $\sigma$ as a product of disjoint cycles as follows: $\left(n_{11}^{\sigma} \cdots n_{1 l_{1}}^{\sigma}\right)\left(n_{21}^{\sigma} \cdots n_{2 l_{2}}^{\sigma}\right) \cdots\left(n_{r 1}^{\sigma} \cdots n_{r l_{r}}^{\sigma}\right)$ with $n_{i 1}^{\sigma}<n_{i j}^{\sigma}$ for all $i \in[r]$ and $j \in\left[l_{r}\right] \backslash\{1\}$ and satisfying $n_{11}^{\sigma}>n_{21}^{\sigma}>\cdots>n_{r 1}^{\sigma}$. Let $w_{\sigma}$ denote the monomial $x_{n_{11}^{\sigma}, n_{12}^{\sigma}} \cdots x_{n_{1 l_{r}}^{\sigma}, n_{11}^{\sigma}} \cdots x_{n_{r 1}^{\sigma}, n_{r 2}^{\sigma}} \cdots x_{n_{r l_{r}}^{\sigma}, n_{r 1}^{\sigma}}$.

Let $C_{n}$ denote the set of all 1-cycles in $S_{n}$, i.e permutations whose cycle decomposition consists of a single cycle of length $n$. Define the polynomial $\operatorname{HC}_{n}\left(x_{11}, \ldots, x_{n n}\right) \in \mathbb{F}\langle X\rangle$ to be $\sum_{\sigma \in C_{n}} w_{\sigma}$. Fix any directed graph $G$ on $n$ vertices with adjacency matrix $A$. Let $H(G)$ denote $\mathrm{HC}_{n}(A(1,1), \ldots, A(n, n))$. The quantity $H(G)$ has a simple description: if $\mathbb{F}$ is of characteristic 0 , then $H(G)$ is the number of directed Hamiltonian cycles in $G$; and if $\mathbb{F}$ is of characteristic $k$, then $H(G)$ is the number of directed Hamiltonian cycles of $G$ modulo $k$.

We have the following easy lemma:

Lemma 21. There are ABPs $P_{1}^{\prime}$ and $P_{2}^{\prime}$ of size $O\left(n^{2}\right)$ and width $n$ that compute homogeneous polynomials $F_{1}^{\prime}, F_{2}^{\prime} \in \mathbb{F}\langle X\rangle$ of degree $n$ such that for any $\sigma \in S_{n}$, we have 
- $F_{1}^{\prime}\left(w_{\sigma}\right)=\operatorname{sgn}(\sigma)$.

- $F_{2}^{\prime}\left(w_{\sigma}\right)=\operatorname{sgn}(\sigma)$ if $\sigma \in C_{n}$ and 0 otherwise.

Moreover, the above ABPs can be computed in time poly $(n)$.

Proof. Recall that given a permutation $\sigma \in S_{n}$, the quantity $\operatorname{sgn}(\sigma)$ is $(-1)^{n+c_{\sigma}}$, where $c_{\sigma}$ is the number of disjoint cycles in $\sigma$. Moreover, note that if $\sigma$ as a product of disjoint cycles is $\left(n_{11}^{\sigma} \cdots n_{1 l_{1}}^{\sigma}\right)\left(n_{21}^{\sigma} \cdots n_{2 l_{2}}^{\sigma}\right) \cdots\left(n_{r 1}^{\sigma} \cdots n_{r l_{r}}^{\sigma}\right)$ as above, the value $c_{\sigma}$ is simply the number of left-to-right minima in this representation, i.e the number of $n_{i j}^{\sigma}$ such that $n_{i j}^{\sigma}<n_{k l}^{\sigma}$ for all $n_{k l}^{\sigma}$ to the left of $n_{i j}^{\sigma}$. Using this observation, it is easy to design an ABP $P_{1}^{\prime}$ that keeps track of the sign of the permutation and computes a polynomial $F_{1}^{\prime}$ as above. The ABP $P_{2}^{\prime}$ can be constructed similarly; the main difference from the case of $P_{1}^{\prime}$ is that the ABP must produce the coefficient 0 unless $n_{11}^{\sigma}=1$. We omit the formal description of $P_{1}^{\prime}$ and $P_{2}^{\prime}$.

The analogue of Theorem 10 for the Moore determinant follows below. The statement here is stronger: we show that the arithmetic circuit complexity of $\operatorname{Mdet}_{n}(X)$ is polynomial if and only if the arithmetic circuit complexity of $\operatorname{Mperm}_{n}(X)$ is polynomial.

Theorem 22. The Moore determinant polynomial $\operatorname{Mdet}_{n}(X)$ can be computed by a polynomialsized noncommutative arithmetic circuit if and only if the Moore permanent polynomial $\operatorname{Mperm}_{n}(X)$ can be computed by a polynomial-sized noncommutative arithmetic circuit.

Proof. As in the proof of Theorem 10, we will use the Hadamard product; this time, it can be used to erase or introduce the signs of the permutations corresponding to each monomial $w_{\sigma}$. Formally, we have $\operatorname{Mperm}_{n}(X)=\operatorname{Mdet}_{n}(X) \circ F_{1}^{\prime}(X)$ and $\operatorname{Mdet}_{n}(X)=\operatorname{Mperm}_{n}(X) \circ F_{1}^{\prime}(X)$, where $F_{1}^{\prime}(X)$ is the polynomial defined in the statement of Lemma 21. Hence, if $\operatorname{Mdet}_{n}(X)\left(\operatorname{resp} . \operatorname{Mperm}_{n}(X)\right)$ is computed by a noncommutative arithmetic circuit of size $s$, then by applying Corollary 4 , we see that $\operatorname{Mperm}_{n}(X)\left(\operatorname{resp} . \operatorname{Mdet}_{n}(X)\right)$ is computed by a noncommutative arithmetic circuit of size $\operatorname{poly}(s, n)$.

Remark 23. Note that Theorem 20 proves an equivalence (up to polynomial factors) between the arithmetic circuit complexities of the Moore determinant and permanent. This is a stronger statement than we obtained in the case of the Cayley determinant and permanent, where we only showed (roughly) that the Cayley determinant is at least as hard to compute as the Cayley permanent. The reason for this is that we are unable to obtain a small $A B P$ that performs the function of $P_{1}^{\prime}$ for the monomials $m_{\sigma}$ (defined in Section 4 ): that is, a small ABP computing a polynomial $F_{1}$ such that $F_{1}\left(m_{\sigma}\right)=\operatorname{sgn}\left(m_{\sigma}\right)$ for every $\sigma \in S_{n}$. However, we are unable to rule out the possibility that such an $A B P$ exists. If it does, then as above, we can obtain a simple equivalence between the complexities of the Cayley determinant and permanent.

We now consider the complexity of computing the Moore determinant over matrix algebras of polynomial dimension. We can, as in the previous sections, show that this is at least as hard as computing the permanent over matrices with entries from $\mathbb{F}$, but we take a different route this time. We show that if the Moore determinant over a field of characteristic $k$ can be computed 
in polynomial time, then there is a polynomial-time algorithm to compute the number of directed Hamilton cycles $H(G)$ modulo $k$ for an input directed graph $G$. This allows us to draw stronger consequences, namely that the Moore determinant is hard to compute even when the field $\mathbb{F}$ is of characteristic 2, something that would not follow if we reduced the permanent to this problem (since the permanent is polynomial-time computable over fields of characteristic 2).

Theorem 24. If there is a polynomial-time algorithm $\mathcal{A}$ that computes the $n \times n$ Moore determinant of matrices with entries in $M_{S}(\mathbb{F})$, for $S=c \cdot n^{2}$ for suitable $c>0$, then there is a polynomial-time algorithm that, on input a directed graph $G$, computes $H(G)$.

Proof. Note that $\mathrm{HC}_{n}(X)=\operatorname{Mdet}_{n}(X) \circ F_{2}^{\prime}$, where $F_{2}^{\prime}$ is the polynomial computed by ABP $P_{2}^{\prime}$ constructed in Lemma 21, Moreover, $H(G)=\mathrm{HC}_{n}(A(1,1), \ldots, A(n, n))$, where $A$ is the adjacency matrix of the graph $G$. Hence, to compute $H(G)$, we need to compute $\mathrm{HC}_{n}(A(1,1), \ldots, A(n, n))$, which can be done in polynomial time by Corollary 5 .

\section{Completeness Results}

In this section we observe that the noncommutative Cayley determinant over integer matrices is complete for GapP w.r.t. polynomial-time Turing reductions. Likewise, the noncommutative Cayley determinant over a finite field of characteristic $k \neq 2$ is hard for the modular counting complexity class $\operatorname{Mod}_{k} \mathrm{P}$ w.r.t. polynomial-time Turing reductions. These observations also hold for the symmetrized determinant. For the Moore determinant, we prove the above results without any restriction on the characteristic of the underlying field. We formally describe these observations.

Definition 25. FFK94, BG92 A function $f: \Sigma^{*} \longrightarrow \mathbb{Z}$ is in GapP if there is a polynomial time NDTM $M$ such that for each $x \in \Sigma^{*}$ the value $f(x)$ is $\operatorname{acc}_{M}(x)-\operatorname{rej}_{M}(x)$.

For a prime $k$, the class $\operatorname{Mod}_{k} \mathrm{P}$ consist of languages $L \subseteq \Sigma^{*}$ such that for some function $f \in \operatorname{GapP}$ we have $x \in L$ if and only if $f(x) \equiv 0(\bmod k)$.

By Valiant's result [V79] it is known that the integer permanent is GapP-complete with respect to polynomial-time Turing reductions. Furthermore, the permanent over $\mathbb{F}_{k}$ is $\operatorname{Mod}_{k} \mathrm{P}$-hard for prime $k \neq 2$.

Now, for $n \in \mathbb{N}$, consider the Cayley determinant for $2 n \times 2 n$ matrices with entries from $M_{S}(\mathbb{Z})$, where $S=c n^{2}$ for some constant $c$. By Theorem 11, there is a fixed $c>0$ such that computing the integer permanent for $n \times n$ matrices is polynomial-time reducible to computing the $(1, S)^{t h}$ entry of such a Cayley determinant. The same observation holds modulo $k$ for a prime $k$.

Furthermore, the problem of computing the $(1, S)^{t h}$ entry of such a Cayley determinant over $\mathbb{Z}$ is easily seen to be in GapP: we can design a polynomial-time NDTM which takes as input a $2 n \times 2 n$ matrix with entries from $M_{S}(\mathbb{Z})$ and the difference in the number of accepting and rejecting paths is the $(1, S)^{t h}$ entry of its Cayley determinant. Hence we have the following. 
Corollary 26. There exists a constant $c$ such that the following holds. For $S=c n^{2}$, computing the $(1, S)^{t h}$ entry of the Cayley determinant for $2 n \times 2 n$ matrices with entries from $M_{S}(\mathbb{Z})$ is GapPcomplete w.r.t. polynomial-time Turing reductions. Given a finite field $\mathbb{F}$ of characteristic $k \neq 2$, computing the $(1, S)^{\text {th }}$ of the Cayley determinant for $2 n \times 2 n$ matrices over $M_{S}(\mathbb{F})$ is hard w.r.t. polynomial-time Turing reductions for $\operatorname{Mod}_{k} \mathrm{P}$.

We have similar GapP-completeness and $\operatorname{Mod}_{k} \mathrm{P}$-hardness consequences for the symmetrized determinant from the results in Sections 6. For the Moore determinant, by Theorem 24, we additionally obtain hardness for $\oplus \mathrm{P}$ over fields of characteristic 2 .

Corollary 27. There exists a constant $c$ such that the following holds. For $S=c n^{2}$, computing the $(1, S)^{\text {th }}$ entry of the Moore determinant for $2 n \times 2 n$ matrices with entries from $M_{S}(\mathbb{Z})$ is GapPcomplete w.r.t. polynomial-time Turing reductions. Given a finite field $\mathbb{F}$ of any characteristic $k>1$, computing the $(1, S)^{t h}$ of the Moore determinant for $2 n \times 2 n$ matrices over $M_{S}(\mathbb{F})$ is hard w.r.t. polynomial-time Turing reductions for $\operatorname{Mod}_{k} \mathrm{P}$.

Proof. The result follows from Theorem 24 and the following observations: computing $H(G)$ over the rationals on an input graph $G$ is GapP-complete w.r.t. polynomial-time Turing reductions; similarly, computing $H(G)$ over a field $\mathbb{F}$ of characteristic $k$ (including $k=2$ ) is hard for $\operatorname{Mod}_{k} \mathrm{P}$ w.r.t. polynomial-time Turing reductions.

\section{Discussion}

Our work raises further interesting questions regarding the complexity of the noncommutative determinant.

An important open question is the complexity of computing the noncommutative determinant over constant dimensional matrix algebras. Theorem 11 can be easily used to show that assuming that the permanent of an $n \times n$ matrix over $\mathbb{F}$ cannot be computed in subexponential time, the $n \times n$ noncommutative Cayley, symmetrized, and Moore determinants with entries from $M_{(\log n)^{\omega(1)}}(\mathbb{F})$ cannot be computed in polynomial time. Can one strengthen this result to one that says something about computing the Cayley or Moore determinant over matrices with entries from $M_{c}(\mathbb{F})$ for some absolute constant $c$ ? (Recall that the symmetrized determinant, on the other hand, is efficiently computable over constant dimensional matrix algebras.) It is interesting to note that [CS04] have shown an exponential lower bound for the ABP complexity of the Cayley determinant over even $2 \times 2$ matrices.

A question that arises from the results of Section 4 is if one can show - analogous to those results - that the Cayley permanent is at least as hard to compute as the Cayley determinant. As pointed out in Remark 23, one way to prove this is to construct a small ABP $P_{1}$ that computes a polynomial $F_{1}$ of degree $n$ such that $F\left(x_{1, \sigma(1)} \ldots x_{n, \sigma(n)}\right)=\operatorname{sgn}(\sigma)$ for every $\sigma \in S_{n}$. This would also make the proofs of Theorems 10 and 11 much more transparent.

Finally, note that our results do not imply that the Cayley determinant is hard to compute over $M_{k}(\mathbb{F})$ when $\mathbb{F}$ is a field of characteristic 2 , since the permanent is known to be polynomial-time 
computable over such fields. On the other hand, we have proved that the Moore determinant over such domains (where $k$ is polynomial) is hard for $\oplus \mathrm{P}$. Can we prove an analogous result for the Cayley determinant?

\section{References}

[AJS09] V. Arvind, P. S. Joglekar, S. Srinivasan. Arithmetic Circuits and the Hadamard Product of Polynomials CoRR abs/0907.4006: (2009). http://arxiv.org/abs/0907.4006. In Proceedings FSTTCS 2009 conference, December 2009, to appear.

[A96] H. Aslaksen. Quaternionic determinants. Math. Intelligencer 18 (1996), no. 3, 57-65.

[B] A. Barvinok. New Permanent Estimators via Non-Commutative Determinants. preprint available from http://www.math.lsa.umich.edu/ barvinok/papers.html

[BG92] R. Beigel, J. Gill. Counting Classes: Thresholds, Parity, Mods, and Fewness. Theor. Comput. Sci. 103(1): 3-23 (1992).

[CS04] S. Chien, A. Sinclair. Algebras with polynomial identities and computing the determinant In Proc. Annual IEEE Sym. on Foundations of Computer Science,352-361, 2004.

[CRS03] S. Chien, L. E. Rasmussen, A. Sinclair. Clifford algebras and approximating the permanent. J. Comput. Syst. Sci. 67(2): 263-290 (2003).

[FFK94] S. A. Fenner, L. Fortnow, S. A. Kurtz. Gap-Definable Counting Classes. J. Comput. Syst. Sci. 48(1): 116-148 (1994).

[GG81] C. Godsil, I. Gutman. On the matching polynomial of a graph, Algebraic Methods in Graph Theory, 1981, pp. 241-249.

[KKL+93] N. Karmarkar, R. M. Karp, R. J. Lipton, L. Lovàsz, Michael Luby. A MonteCarlo Algorithm for Estimating the Permanent. SIAM J. Comput. 22(2): 284-293 (1993).

[LS09] D. Lundholm, L. Svensson. Clifford algebra, geometric algebra, and applications. Available at http://arxiv.org/abs/0907.5356

[MR09] C. Moore, A. Russell. Approximating the Permanent via Nonabelian Determinants, CoRR abs/0906.1702: (2009). http://arxiv.org/abs/0906.1702

[N91] N. NisAn. Lower bounds for noncommutative computation In Proc. of 23rd ACM Sym. on Theory of Computing, 410-418, 1991.

[RS05] R. RAz, A. ShPILKA. Deterministic polynomial identity testing in non commutative models Computational Complexity,14(1):1-19, 2005.

[V79] L. G. Valiant. The Complexity of Computing the Permanent. Theor. Comput. Sci. 8: 189-201 (1979). 